\title{
Characteristics of COVID-19 Clinical Trials in China Based on the Registration Data on ChiCTR and ClinicalTrials.gov
}

This article was published in the following Dove Press journal:

Drug Design, Development and Therapy

\author{
Jihan Huang $\mathbb{D}^{1, *}$ \\ Yingchun $\mathrm{He}^{1, *}$ \\ Qianmin $\mathrm{Su}^{2}$ \\ Juan Yang (iD)
}

'Center for Drug Clinical Research, Institute of Interdisciplinary Integrative Medicine Research, Shanghai University of Traditional Chinese Medicine, Shanghai 201203, People's Republic of China; ${ }^{2}$ Department of Computer in College of Electronic and Electrical Engineering, Shanghai University of Engineering Science, Shanghai 201620, People's Republic of China

*These authors contributed equally to this work
Correspondence: Jihan Huang; Juan Yang

Center for Drug Clinical Research, Institute of Interdisciplinary Integrative Medicine Research, Shanghai University of Traditional Chinese Medicine, \#1200 Cailun Road, Pudong New District, Shanghai 201203, People's Republic of China

Tel +86 2I 5I322420

Email huangjihan@shutcm.edu.cn; janeyang5006@I63.com
Objective: This study aimed to evaluate the fundamental characteristics of coronavirus disease (COVID-19) clinical trials registered in China.

Methods: COVID-19 clinical trials registered in China were analyzed from databases on ChiCTR and ClinicalTrials.gov. The study designs, samples, primary end points, and intervention measures were evaluated.

Results: In total, 262 intervention clinical trials were retrieved on March 10, 2020. Overall, $181(69.1 \%)$ trials involved two groups, 200 (76.3\%) trials were randomized parallel trials, $24(9.2 \%)$ trials were double blind, and $60.3 \%$ of trials included $\leq 100$ participants. Sixty (22.9\%) trials considered symptom improvement as the primary endpoint and $43(16.4 \%)$ trials considered the rate or time at which the subjects became virus-free as the primary endpoint. Of 262 intervention studies, chemical drugs and biological products were studied in $105(40.1 \%)$ intervention studies, of which antiviral drugs accounted for $15.3 \%$ and malaria drugs accounted for $8.4 \%$ of the studies. Among all trials, $27.9 \%$ of the studies used traditional Chinese medicine (TCM), 10.3\% used cell therapy, and 5.0\% used plasma therapy.

Conclusion: This study is the first snapshot of the landscape of COVID-19 clinical trials registered in China and provided the basic features of clinical trial designs for the treatment and prevention of COVID-19 to offer useful information to guide future clinical trials on COVID-19 in other countries.

Keywords: COVID-19, clinical trial, interventional, randomized, blinding

\section{Introduction}

Recently, coronavirus disease (COVID-19) ${ }^{1}$ emerged in Wuhan, Hubei Province, China, and has since become a pandemic. Currently, the situation in many countries such as South Korea, Italy, and Iran is critical. The most common symptoms of COVID-19 are fever and cough. ${ }^{2}$ In severe cases, the infection can cause pneumonia, severe acute respiratory syndrome, kidney failure, and even death. The mortality in the elderly (ie, over 65 years old) with underlying diseases is high. The coronaviruses belong to a new RNA virus strain from the Coronaviridae family, ${ }^{3}$ and SARS-CoV-2 is the seventh member of the coronavirus family that infects humans. ${ }^{4}$ At present, there are no effective antiviral drugs confirmed for treating COVID-19; and symptomatic support and comprehensive intervention are the main treatment methods. However, treatment with the traditional Chinese medicine (TCM) and the combination of TCM and Western medicine have produced 
satisfactory therapeutic effects in China and Vellingiri et al also suggested that traditional Indian medicinal plants may be a novel treatment for COVID-19. ${ }^{5} \mathrm{Lu}$ reviewed the possible treatments for COVID-19, and stated the safety and effectiveness of such treatments remain to be confirmed. ${ }^{6}$ Additionally, Iyer et al have reviewed efficient diagnostics, and therapeutic approaches for COVID-19. ${ }^{7}$

In medical research, clinical trials are essential for promoting human health. Clinical trials on new drugs and interventions are registered to provide the public, health professionals, researchers, and sponsors with reliable information, rendering the design and implementation of clinical trials transparent, and enabling the search and evaluation of such trials on the internet. ${ }^{8,9}$ People can retrieve crucial information about the initial stages of the trials from clinical trial registries, and learn from the methods used to conduct them. Registering clinical trials also facilitates international cooperation and enables sponsors to use funds in meaningful ways. In addition, registration prevents researchers from making biased decisions because of publication bias or incomplete reports, wherein, the negative results are withheld or ambiguous results have been presented. ${ }^{10,11}$

ClinicalTrials.gov is the largest registry of clinical trials, which has been established and is operated by the US National Institutes of Health and the US National Library of Medicine. All types of clinical trials conducted worldwide can be registered in advance on ClinicalTrials. gov. Besides, this website enables users to search for all ongoing international multicenter clinical trials. ${ }^{12}$ By the end of 2019, 327,525 clinical trials had been registered on ClinicalTrials.gov, with 209 participating countries. The Chinese Clinical Trial Registry (ChiCTR) was established in 2005 and was recognized as the representative registry of China to be a part of the World Health Organization International Clinical Trials Registry Platform in 2007. The ChiCTR has the highest number of registered clinical trials than any other clinical trial network in China. Based on the registered clinical trial data, we have successfully researched for the sample sizes for clinical trials investigating the dosage, ${ }^{13}$ reviewed the clinical trials on ginseng, ${ }^{14}$ and studied the characteristics of TCM clinical trials. ${ }^{15}$ Although Zhang et al analyzed the clinical trials, their study period ended on February 12, 2020, and only 87 intervention trials were studied. ${ }^{16}$

The number of COVID-19 clinical trials registered with ClinicalTrials.gov and ChiCTR has grown considerably over the past 3 months. In the present study, the content from clinical trials registered in China was summarized and analyzed from various perspectives as a reference for the design of clinical trials for COVID-19.

\section{Materials and Methods}

\section{Search Strategy}

"COVID-19" and "China" were used as the keywords to search for all COVID-19 clinical trials registered from China on ClinicalTrials.gov and ChiCTR on March 10, 2020. All retrieved records were downloaded, and the following data were collected: ChiCTR or ClinicalTrials. gov identifier, study title, study type, study design, primary outcome measures, condition, number of groups, and sample size. A standard Microsoft-Excel database was created for the analysis.

\section{Statistical Analyses}

All continuous values are expressed as median and interquartile range, and categorical variables are expressed as percentages. All statistical procedures were performed using the SAS software (v9.4; SAS Institute, Cary, NC, USA).

\section{Results}

\section{Number of COVID-19 Clinical Trials Registered in China}

In total, 429 COVID-19 clinical trials were registered from China (359 in ChiCTR and 70 in ClinicalTrials.gov). After the exclusion of 127 observational trials and 40 other trials based on basic science, epidemiology, health services, prevention, prognosis, diagnosis, and treatment studies; 262 clinical trials were defined as interventional clinical trials (Figure 1 and Supplementary data file).

\section{General Analysis of Interventional Clinical Trials}

Interventional clinical trials, stratified by the study design, were as follows: randomized parallel, 200 (76.3\%); nonrandomized parallel, 21 (8.0\%); and single-group assignment, $41(15.6 \%)$. The clinical trials according to the type of blinding were as follows: double-blind, 24 (9.2\%); single-blind, 12 (4.6\%); open-label, 96 (36.6\%); and not provided, 130 (49.6\%).

Overall, 35 trials involved a single group (13.4\%), 181 trials involved two groups (69.1\%), 32 trials involved three groups $(12.2 \%)$, and 14 trials involved at least four groups $(5.4 \%)$. The clinical trials stratified by the severity 


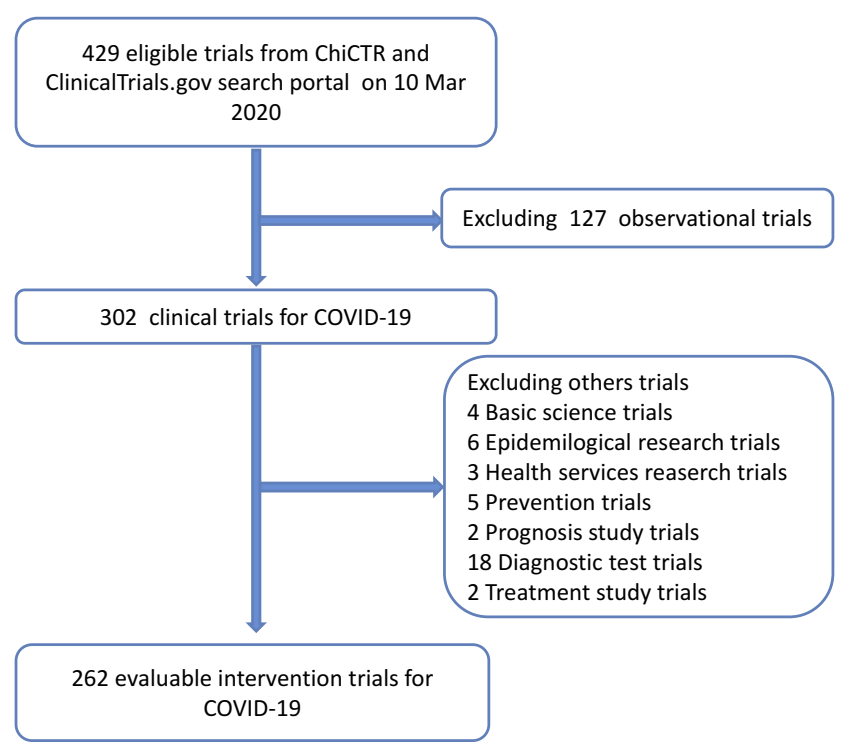

Figure I Flowchart of screening process and search results. Adapted from $\mathrm{He} \mathrm{Y}$, Yang J, Lv Y, et al. A review of ginseng clinical trials registered in the WHO international clinical trials registry platform. Biomed Res Int. 2018;2018:184314. Creative Commons license and disclaimer available from: http://creativecommons. org/licenses/by/4.0/legalcode. ${ }^{14}$

of symptoms were as follows: mild and moderate, 213 (81.3\%); and severe and critical, 49 (18.7\%) (Table 1).

The sample size among the 262 intervention trials was between 9 and 1000 cases, and the cumulative statistical sample size was 36,406 cases, of which the minimum sample size was 9 cases (ChiCTR2000030300). Two clinical trials had the largest sample size of 1000 cases (ChiCTR2000030386 and ChiCTR2000029578), and were the TCM clinical trials.

In summary, $60.3 \%$ of the trials included $\leq 100$ participants, $35.5 \%$ of trials involved $100-400$ participants, and $4.2 \%$ involved $>400$ participants (Figure 2).

\section{Primary Outcome Measures of Interventional Clinical Trials}

In most clinical trials, symptom improvement was the primary outcome measure, which included the symptom improvement rate and recovery time. COVID-19 is an infectious disease caused by a virus, and hence, the negative effects of the virus are the key indicators for identifying a cure for this disease. The mortality rates are higher in the elderly and critically ill patients than in the young subjects; however, some trials have used mortality as the primary endpoint.

When classifying the studies by primary outcome measures, $22.9 \%$ studies focused on the improvement in clinical symptoms, $16.4 \%$ on the rate or time at which the subjects became virus-free, $11.8 \%$ on the change in pneumonia severity,
Table I Trial Characteristics and Summary of Designs for All Interventional Trials $(n=262)$

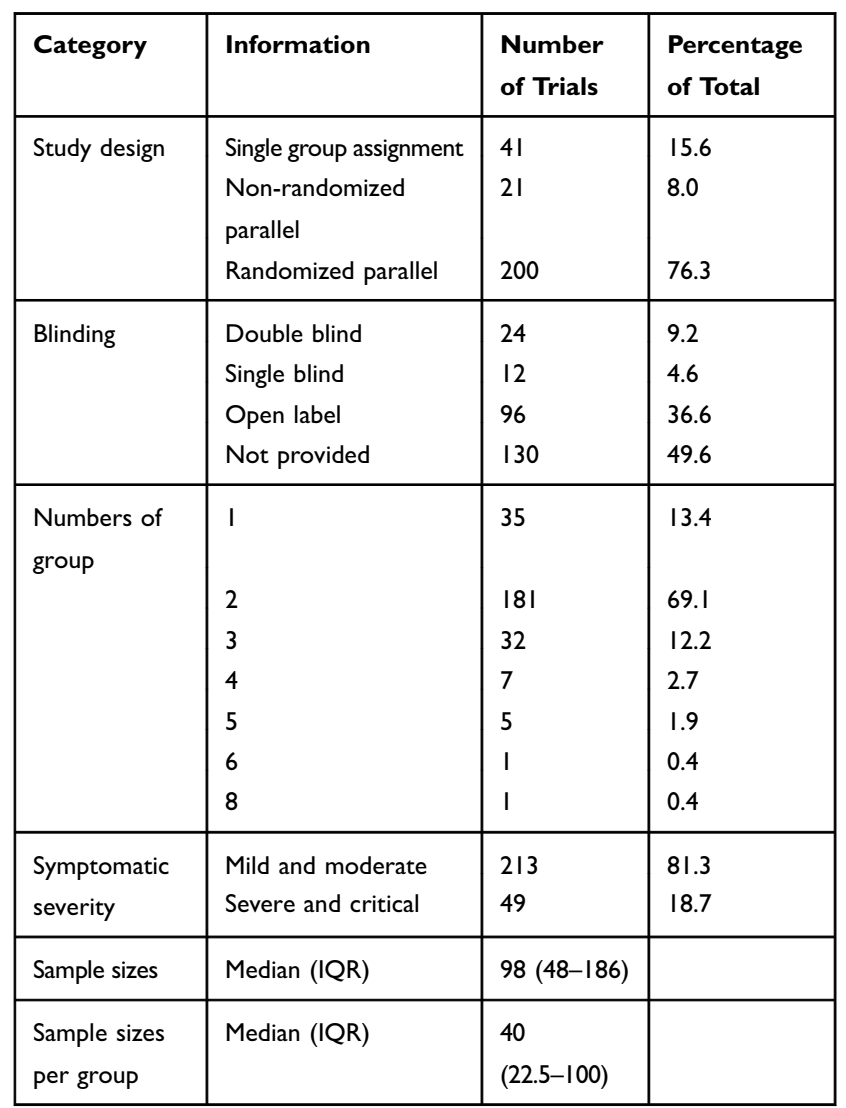

$6.9 \%$ on the time required for a fever to subside, $6.5 \%$ on the cure rate, and $5.7 \%$ on the mortality rate (Table 2 ).

\section{Intervention Protocol Type Analysis}

The intervention protocols used in the studies are presented in Table 3. Among the 262 clinical trials, $40.1 \%$

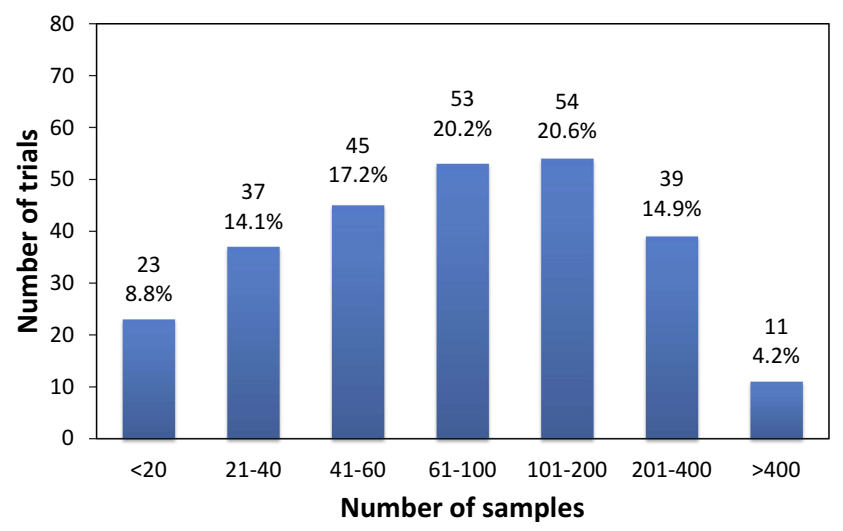

Figure 2 Distribution of sample sizes in all interventional trials on COVID-19 in China. Copyright @20I5. Dove Medical Press. Adapted from Huang J, Su Q, Yang J, et al. Sample sizes in dosage investigational clinical trials: a systematic evaluation. Drug design, development and therapy. 2015; 9:305-312. ${ }^{13}$ 
Table 2 Primary Outcome Measures Covered in the Included Trials $(n=262)$

\begin{tabular}{|l|l|l|}
\hline Category & $\begin{array}{l}\text { Number } \\
\text { of Trials }\end{array}$ & $\begin{array}{l}\text { Percentage of } \\
\text { Total }\end{array}$ \\
\hline Virus negative conversion rate or time & 43 & 16.4 \\
Fever to normal time & 18 & 6.9 \\
Mortality rate & 15 & 5.7 \\
Time to clinical recovery & 60 & 22.9 \\
In-hospital time & 11 & 4.2 \\
The change of pneumonia severity & 31 & 11.8 \\
TCM symptom score & 4 & 1.5 \\
Cure rate & 17 & 6.5 \\
Oxygen saturation & 17 & 6.5 \\
Questionnaire or scale & 15 & 5.7 \\
The incidence of side effects & 12 & 4.6 \\
Others & 19 & 7.3 \\
\hline
\end{tabular}

Table 3 Interventions Covered in the Included Trials $(n=262)$

\begin{tabular}{|l|l|l|}
\hline Category & $\begin{array}{l}\text { Number } \\
\text { of Trials }\end{array}$ & $\begin{array}{l}\text { Percentage } \\
\text { of Total }\end{array}$ \\
\hline Chemical or Biological & 105 & 40.1 \\
Antivirals & 40 & 15.3 \\
Antimalarials & 22 & 8.4 \\
Antineoplastic and immunomodulating agents & 25 & 9.5 \\
Glucocorticoids & 5 & 1.9 \\
Mucolytics & 2 & 0.8 \\
Other & 11 & 4.2 \\
Traditional Chinese medicine or integrated & 73 & 27.9 \\
medicine & & \\
Cell therapy & 27 & 10.3 \\
Behavioral intervention & 13 & 5.0 \\
Plasma & 13 & 5.0 \\
Psychological intervention & 9 & 3.4 \\
Medical instruments & 7 & 2.7 \\
Acupuncture and massage & 4 & 1.5 \\
Vaccine & 3 & 1.1 \\
Other & 8 & 3.1 \\
\hline Total & 262 & 100 \\
\hline
\end{tabular}

used chemical or biological products accounted, among which, $15.3 \%$ involved antiviral medications, and $8.4 \%$ involved antimalarial medications. TCM or integrated therapy, cell therapy, and plasma therapy accounted for $27.9 \%, 10.3 \%$, and $5.0 \%$, respectively, of the clinical trials on chemical or biological products. A COVID-19 vaccine developed by the Shenzhen Genoimmune Medical Institute (Shenzhen, china) is undergoing clinical trials with a sample size of 200 cases. The registration numbers of the clinical trials are NCT04299724 and NCT04276896.

\section{Discussion}

The COVID-19 outbreak has been listed by the World Health Organization as a major public health emergency of international concern that seriously endangers public health and safety. No effective antiviral therapy has been identified yet for COVID-19. Symptomatic supportive therapy and comprehensive intervention are the main treatment methods for COVID-19. During the pandemic, frontline medical staff are required to constantly improve the treatment methods; therefore, many clinical trials are necessary. Clinical trial registration is the primary link ensuring clinical trial transparency, crucial content for medical research ethics, and researchers' responsibility and obligations. As the research on prevention and control of COVID-19 progresses, the number of registered clinical trials has continued to grow. As of March 10, 2020, 429 clinical trials for COVID-19 have been conducted in China.

Operating double-blinded trials in the current emergency COVID-19 situation is difficult, particularly for the cell therapy and other behavioral intervention trials. However, regarding the design type, the proportion of randomized controlled trials remains the largest, because this design type has the potential of causing the least the bias, and the level of evidence is the highest in clinical studies. ${ }^{17}$ Without any reliable evidence, single-arm trials are an acceptable trial design. ${ }^{18}$ Although single-arm tests can be used before and after the control, single-arm trials are closer to clinical practice in operation. Most retrieval trials are exploratory, and their sample size is not supposed to be too large. ${ }^{13}$ Thus, the expected sample size can be completed in a short time, and the trial results can be obtained faster. These clinical trials were conducted with the main features of the current outbreak; therefore, they should be accepted during this pandemic period. The clinical trial design must adhere to the basic principles of randomization, control, and replication; use objective primary endpoint, and blind evaluation as far as possible; and follow statistical guidelines for clinical trials to avoid hasty analysis leading to incorrect conclusions.

The currently registered studies have had many primary endpoints, including improvement of clinical symptoms, time or rate of becoming viral-negative, and mortality. Given the high cure rate of the disease, improving symptoms is critical for reducing the critical illness rate and mortality. These indicators of efficacy can be evaluated in the near future. As a crucial discharge 
standard, the rate at which the subjects become virus-free represent the recovery rate of the patients and the duration infected with the virus, respectively. The absence of detectable virus nucleic acid can reduce the spread of the virus. Because the mortality rate of mild to moderate patients with this disease is low, the mortality rate is suitable as the primary endpoint for severe patients.

Although no effective therapy has been developed for COVID-19, various intervention measures have been adopted, including TCM, antiviral drugs, cell therapy, plasma therapy, behavioral intervention, and psychological intervention. In the retrieved studies, even with the administration of antiviral therapy, further interventions were still required. The antiviral drugs included azvudine, Ganovo/ritonavir, danoprevir, favipiravir, ritonavir/ritonavir, baloxavir marboxil, remdesivir, oseltamivir, Arbidol, ritonavir, darunavir/cobicistat, and lopinavir/ritonavir. These antiviral drugs are potentially effective for treating COVID-19., ${ }^{6,19}$ A trial using favipiravir, an antiinfluenza drug used to treat life-threatening RNA viral infections, ${ }^{20}$ has been carried out (registration number chictr20000029600), and the results will soon be published.

Remdesivir is an investigational antiviral drug developed by Gilead Sciences (Foster City, CA, USA) to treat COVID-19 and Ebola. ${ }^{21}$ Holshue et al reported the entire diagnosis and treatment process of the first confirmed patient in the United States, and the patient's symptoms improved after remdesivir use as an emergency treatment. ${ }^{22}$ At present, two clinical trials (NCT04252664 and NCT04257656) of remdesivir in China have attracted much attention. Both trials were randomized, double-blind, and placebo-controlled studies. One trial involved 308 mild to moderate COVID-19 patients, and the other involved 453 severe COVID-19 patients. Under the registration number NCT04257656, 453 severe COVID-19 patients were scheduled for admission. With the outbreak under control in China, 237 cases (remdesivir group; 158 and placebo group; 79) were enrolled in the trial, and the study results showed no significant clinical benefit among adult patients admitted for severe COVID-19 treated with remdesivir. $^{23}$ Also, antimalarial drugs potentially treat COVID-19. ${ }^{24}$ The results by Gautret et al showed that hydroxychloroquine is significantly associated with virus elimination in COVID-19 patients and its effect is reinforced by azithromycin. ${ }^{25}$

A limitation of the present study is that the results of the clinical trials were not included. As viral spread is currently being controlled, the number of patients will greatly decrease in China; thus, meeting the needs of all patients registered in clinical trials may be difficult. As the current pandemic worsens, the more we will have to adhere to the evidencebased medicine, for the recovery of the patients, and the effective prevention, and control of the pandemic. At this time, it is necessary for the national and local administrative departments to strengthen the management. The relevant departments should organize research in a scientific, standardized and orderly manner, and call on the relevant departments to conduct on-site inspection, supervision and research. In future research, we will analyze the safety and effectiveness of COVID-19 clinical trials in China and other countries. The efficacy and safety of these clinical trials can provide strong evidence for other countries to fight against COVID-19.

\section{Conclusion}

Based on the registration data obtained from ChiCTR and ClinicalTrials.gov in China, this study revealed the basic characteristics of anti-COVID-19 trials in China including the study designs, samples, primary end points, and intervention measures. This study also provides useful information to guide future clinical trials on COVID-19 in other countries.

\section{Acknowledgments}

We would like to thank all researchers who registered for clinical trials and pay tribute to all the medical staff struggling in the front lines against COVID-19. This study was supported by Shanghai S\&T Innovation Plan (17401970900).

\section{Disclosure}

All authors declare no conflicts of interest in this work.

\section{References}

1. Meng L, Hua F, Bian Z. Coronavirus disease 2019 (COVID-19): emerging and future challenges for dental and oral medicine. $J$ Dent Res. 2020;99(5):481-487. doi:10.1177/0022034520914246

2. Guan WJ, Ni ZY, Hu Y, et al. Clinical characteristics of coronavirus disease 2019 in China. $N$ Engl J Med. 2020;382:1708-1720. doi:10.1056/NEJMoa2002032

3. Wu F, Zhao S, Yu B, et al. A new coronavirus associated with human respiratory disease in China. Nature. 2020;579:265-269. doi:10.1038/ s41586-020-2008-3

4. Zhu N, Zhang D, Wang W, et al. A novel coronavirus from patients with pneumonia in China, 2019. N Engl J Med. 2020;382(8):727-733. doi:10.1056/NEJMoa2001017

5. Vellingiri B, Jayaramayya K, Iyer M, et al. COVID-19: a promising cure for the global panic. Sci Total Environ. 2020;725:138277. doi:10.1016/j.scitotenv.2020.138277

6. Lu H. Drug treatment options for the 2019-new coronavirus (2019-nCoV). Biosci Trends. 2020;14:69-71. doi:10.5582/bst.2020. 01020

7. Iyer M, Jayaramayya K, Subramaniam MD, et al. COVID-19: an update on diagnostic and therapeutic approaches. BMB Rep. 2020;53 (4):191-205. doi:10.5483/BMBRep.2020.53.4.080 
8. McCray AT. Better access to information about clinical trials. Ann Intern Med. 2000;133(8):609-614. doi:10.7326/0003-4819-1338-200010170-00013

9. Zarin DA, Tse T. Medicine. Moving toward transparency of clinical trials. Science (New York, NY). 2008;319(5868):1340-1342. doi:10. 1126/science. 1153632

10. Tse T, Williams RJ, Zarin DA. Reporting "basic results" in ClinicalTrials. gov. Chest. 2009;136(1):295-303. doi:10.1378/chest.08-3022

11. Zarin DA, Ide NC, Tse T, Harlan WR, West JC, Lindberg DA. Issues in the registration of clinical trials. JAMA. 2007;297(19):2112-2120. doi:10.1001/jama.297.19.2112

12. Zarin DA, Keselman A. Registering a clinical trial in ClinicalTrials. gov. Chest. 2007;131(3):909-912. doi:10.1378/chest.06-2450

13. Huang JH, Su QM, Yang J, et al. Sample sizes in dosage investigational clinical trials: a systematic evaluation. Drug Des Devel Ther. 2015;9:305-312. doi:10.2147/DDDT.S76135

14. He Y, Yang J, Lv Y, et al. A review of ginseng clinical trials registered in the WHO international clinical trials registry platform. Biomed Res Int. 2018;2018:1843142. doi:10.1155/2018/1843142

15. Chen J, Huang J, Li JV, Lv Y, He Y, Zheng Q. The characteristics of TCM clinical trials: a systematic review of clinicalTrials.gov. Evid Based Complement Alternat Med. 2017;2017:9461415. doi:10.1155/2017/ 9461415

16. Zhang Q, Wang Y, Qi C, Shen L, Li J. Clinical trial analysis of 2019-nCoV therapy registered in China. J Med Virol. 2020;92:540-545. doi:10.1002/jmv.25733

17. Mad P, Felder-Puig R, Gartlehner G. [Randomised controlled trials]. Wien Med Wochenschr. 2008;158(7-8):234-239. German. doi:10.1007/s10354-008-0526-y
18. Grayling MJ, Mander AP. Do single-arm trials have a role in drug development plans incorporating randomised trials? Pharm Stat. 2016;15(2):143-151. doi:10.1002/pst.1726

19. Dong L, Hu S, Gao J. Discovering drugs to treat coronavirus disease 2019 (COVID-19). Drug Discov Ther. 2020;14(1):58-60. doi:10. 5582/ddt.2020.01012

20. Shiraki K, Daikoku T. Favipiravir, an anti-influenza drug against life-threatening RNA virus infections. Pharmacol Ther. 2020;209:107512. doi:10.1016/j.pharmthera.2020.107512

21. Muyembe-Tamfum JJ, Sivahera B, Camara M, et al. A randomized, controlled trial of ebola virus disease therapeutics. $N$ Engl J Med. 2019;381(24):2293-2303. doi:10.1056/NEJMoa1910993

22. Holshue ML, DeBolt C, Lindquist S, et al. First case of 2019 novel coronavirus in the United States. $N$ Engl J Med. 2020;382 (10):929-936. doi:10.1056/NEJMoa2001191

23. Wang Y, Zhang D, Du G, et al. Remdesivir in adults with severe COVID-19: a randomised, double-blind, placebo-controlled, multicentre trial. Lancet. 2020;395:1569-1578. doi:10.1016/s01406736(20)31022-9

24. Colson P, Rolain JM, Lagier JC, Brouqui P, Raoult D. Chloroquine and hydroxychloroquine as available weapons to fight COVID-19. Int J Antimicrob Agents. 2020;55:105932. doi:10.1016/j.ijantimicag. 2020.105932

25. Gautret P, Lagier JC, Parola P. et al. Hydroxychloroquine and azithromycin as a treatment of COVID-19: results of an open-label non-randomized clinical trial. Int $J$ Antimicrob Agents;2020. 105949. doi:10.1016/j.ijantimicag.2020.105949

\section{Publish your work in this journal}

Drug Design, Development and Therapy is an international, peerreviewed open-access journal that spans the spectrum of drug design and development through to clinical applications. Clinical outcomes, patient safety, and programs for the development and effective, safe, and sustained use of medicines are a feature of the journal, which has also been accepted for indexing on PubMed Central. The manuscript management system is completely online and includes a very quick and fair peer-review system, which is all easy to use. Visit http://www. dovepress.com/testimonials.php to read real quotes from published authors. 